\title{
Partially coherent tunneling through a series of barriers: Inelastic scattering versus pure dephasing
}

\author{
Xin-Qi $\mathrm{Li}^{1,2,3}$ and YiJing Yan ${ }^{1,3}$ \\ ${ }^{1}$ Department of Chemistry, Hong Kong University of Science and Technology, Kowloon, Hong Kong SAR, China \\ ${ }^{2}$ National Laboratory for Superlattices and Microstructures, Institute of Semiconductors, Chinese Academy of Sciences, \\ P.O. Box 912, Beijing 100083, China \\ ${ }^{3}$ Center for Theoretical Chemical Physics, Fudan University, Shanghai 200433, China
}

(Received 3 June 2001; revised manuscript received 9 November 2001; published 10 April 2002)

\begin{abstract}
Within the Büttiker dephasing model, the backscattering in the dephasing process is eliminated by setting a proper boundary condition. Explicit expression is carried out for the effective total tunneling probability in the presence of multiple pure dephasing scatterers with partial coherence. The derived formula is illustrated analytically by various limiting cases, and numerically for its application in tunneling through multibarrier systems.
\end{abstract}

DOI: 10.1103/PhysRevB.65.155326

PACS number(s): 73.23. $-\mathrm{b}, 73.40 .-\mathrm{c}$

To simulate the phase-breaking effect in partially coherent transport through a mesoscopic system, Büttiker proposed a conceptually simple model by coupling electronic reservoirs to the conductor. ${ }^{1,2}$ The dephasing reservoir can be thought of as either a fictitious or a real branch voltage probe. Although this approach appears to be purely phenomenological, it, however, can be justified from a microscopic theory with proper approximations, ${ }^{3-5}$ by viewing that both the electron-phonon interactions and the dephasing reservoir can be described by a self-energy function. Owing to its simplicity, the Büttiker dephasing model has received noticeable attention, ${ }^{6,7}$ and has been applied to transport through various mesoscopic systems. ${ }^{8-18}$

Noticeably, in the original work of Büttiker ${ }^{1,2}$ and the later applications mentioned above, in addition to randomizing the electronic phase, the phase-breaking scatterer would also randomize the electronic momentum. Randomization of momentum means backscattering in the dephasing process, thus introducing an additional resistance. This undesired feature has been noticed and analyzed by a few authors, ${ }^{19-22}$ commonly following the idea by coupling two voltage probes to model a single pure dephasing scatterer. In this paper, based on the original work of Büttiker (i.e., using a single reservoir to model a single dephasing scatterer), ${ }^{1,2}$ an explicit expression will be derived for the effective total tunneling probability through a multibarrier mesoscopic system in the presence of multiple pure-dephasing scatterers with arbitrary incoherence strength. The underlying physics and practical application will be illustrated clearly.

In general, consider the tunneling through a series of barriers shown in Fig. 1, where the squares stand for tunnel barriers, and the triangles for dephasing scatterers. They can be described in terms of scattering matrices as follows. For

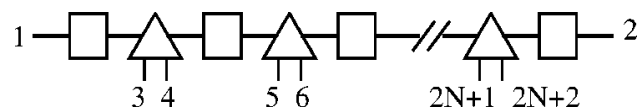

FIG. 1. Partially coherent tunneling through a series of barriers. The square represents a tunnel barrier, and the triangle stands for a phase-breaking scatterer that couples the system to a dephasing reservoir via two channels. the individual (symmetric) barrier (e.g., the $j$ th one), the tunneling property is characterized by ${ }^{23}$

$$
\underline{\mathbf{S}_{j}^{\mathrm{el}}}=\left[\begin{array}{cc}
r_{j} & t_{j} \\
t_{j} & r_{j}
\end{array}\right],
$$

which connects the two incoming amplitudes with the two outgoing amplitudes as is well known. $t_{j}$ and $r_{j}$ are, respectively, the transmission and reflection coefficients through the barrier, and can be characterized by a real parameter $(0$ $\left.\leqslant \delta_{j} \leqslant 1\right)$ as $t_{j}=\sqrt{\delta_{j}}$, and $r_{j}=i \sqrt{1-\delta_{j}}$. For each dephasing scatterer, following Büttiker's approach, ${ }^{1,2}$ a dephasing reservoir is coupled to the system via two channels. During tunneling process, the electron has certain quantum probability of being scattered into the reservoir, undergoing phase randomization in it, then returning into the system via the coupler. As a result of dephasing, the reemitted component does not interfere with that having not entered the reservoir. As a specific model, the coupler is described by the following scattering matrix: ${ }^{1,2}$

$$
\mathbf{S}_{\epsilon}=\left[\begin{array}{cccc}
0 & \sqrt{1-\epsilon} & \sqrt{\epsilon} & 0 \\
\sqrt{1-\epsilon} & 0 & 0 & \sqrt{\epsilon} \\
\sqrt{\epsilon} & 0 & 0 & -\sqrt{1-\epsilon} \\
0 & \sqrt{\epsilon} & -\sqrt{1-\epsilon} & 0
\end{array}\right] .
$$

This $4 \times 4$ matrix connects the four incoming amplitudes with the four outgoing amplitudes along the attached four channels. Here, $\epsilon \in[0,1]$ is a parameter characterizing the incoherence strength ranging from complete coherence to complete incoherence.

To carry out the effective tunneling probability from channel 1 to channel 2 shown in Fig. 1, the $(2 N+2) \times(2 N$ $+2) S$-matrix $\mathbf{S}^{(N)}$ of the entire system is needed. Note that the dimension of $\mathbf{S}^{(N)}$ corresponds to the total number of external channels. $\bar{B} y$ applying either a direct matrix algebra or the Feynman path technique, $\mathbf{S}^{(N)}$ can be carried out via the following recursive expressions: $:^{6,9}$

$$
S_{i I}^{(N)}=\tilde{s}_{i 1}^{(N)}\left[Z_{N} \chi\right] S_{2 I}^{(N-1)},
$$




$$
\begin{gathered}
S_{I i}^{(N)}=S_{I 2}^{(N-1)}\left[Z_{N} \chi\right] \tilde{s}_{1 i}^{(N)}, \\
S_{I J}^{(N)}=S_{I J}^{(N-1)}+S_{I 2}^{(N-1)}\left[Z_{N} \chi \tilde{s}_{11}^{(N)} \chi\right] S_{2 J}^{(N-1)}, \\
S_{i j}^{(N)}=\tilde{s}_{i j}^{(N)}+\tilde{s}_{i 1}^{(N)}\left[Z_{N} \chi S_{22}^{(N-1)} \chi\right] \tilde{s}_{1 j}^{(N)} .
\end{gathered}
$$

Here, $I, J=1,3,4, \ldots, 2 N-1,2 N$, and $i, j=2,2 N+1,2 N+2$; $\mathbf{S}^{(N-1)}$ is the scattering matrix for the subsystem of $N$ tunnel barriers (correspondingly, $N-1$ dephasing scatterers); and the $4 \times 4$ scattering matrix $\widetilde{\mathbf{s}}^{(N)}$ is for the $N$ th single scatterer-

$$
\underline{\mathbf{S}}^{(1)}=Z_{1}\left[\begin{array}{cc}
r_{1}+\alpha^{2} r_{2} & \alpha t_{1} t_{2} \\
\alpha t_{1} t_{2} & r_{2}+\alpha^{2} r_{1} \\
\beta t_{1} & \alpha \beta r_{1} t_{2} \\
\alpha \beta t_{1} r_{2} & \beta t_{2}
\end{array}\right.
$$

Here $\alpha=\sqrt{1-\epsilon}$ and $\beta=\sqrt{\epsilon}$ are introduced to simplify the notation, and $Z_{1}=\left(1-\alpha^{2} r_{1} r_{2}\right)^{-1}$. The most important advantage of the present technique is its ability in treating multiple dephasing scatterers. To this end, we need the $S$ matrix of the added segment of scatterer barrier. It can be easily obtained from Eq. (4) by setting $r_{1}=0$ and $t_{1}=1$, and replacing $t_{2}\left(r_{2}\right)$ by $t_{j}\left(r_{j}\right)$ for the $j$ th coherent barrier. Furthermore, the incoherent parameter $\epsilon$, i.e., $\alpha=\sqrt{1-\epsilon}$ and $\beta=\sqrt{\epsilon}$ in Eq. (4), can also be replaced by $\epsilon_{j}$ for the experimentally avoidable nonuniformity. We denote the resulting $4 \times 4 S$ matrix by $\widetilde{\mathbf{s}}^{(j)}$ [c.f. Eq. (3)]. Using Eq. (4) and the recursive rules of Eq. (3), we can therefore evaluate the $S$ matrix $\mathbf{S}^{(N)}$ for the entire system, thus calculating the effective tunneling probability through the system (i.e., from channels 1 to 2 ). In the following, for the convenience of comparison, we first present result under the momentum randomization boundary condition. Then, the formal result and its implication/application based on the pure dephasing boundary condition are carried out comparatively.

Momentum randomization boundary condition. Under this boundary condition, the reemitting current from the $n$th reservoir into the system is assumed to be equally injected through its $(2 n+1)$ th and $(2 n+2)$ th channels. We denote this current as $\widetilde{J}_{n}$. In the dephasing process, the electron number is conserved, thus the net current of the two channels connecting the system and the reservoir is zero. This feature is characterized by

$$
(2 \underset{\mathbf{I}}{-\widetilde{\mathbf{T}}}) \widetilde{\mathbf{J}}_{B}=\widetilde{\mathbf{K}}^{(1)} J_{L}
$$

where $\mathbf{I}$ is the $N \times N$ unit matrix, and $\widetilde{\mathbf{J}}_{B}$ $=\left(\widetilde{J}_{1}, \widetilde{J}_{2}, \ldots, \widetilde{J}_{N}\right)^{T}$. Hereafter the superscript $(\cdots)^{T}$ means transposition of matrix. $J_{L}$ is the current injected from the left channel 1. $\widetilde{\mathbf{T}}$ is a $N \times N$ matrix with elements $\widetilde{T}_{n m}=T_{2 n+1,2 m+1}+T_{2 n+1,2 m+2}+T_{2 n+2,2 m+1}+T_{2 n+2,2 m+2}$, barrier segment. $Z_{N}=\left[1-\chi S_{22}^{(N-1)} \chi \tilde{s}_{11}^{(N)}\right]^{-1}$, resulting physically from the multiple reflections. ${ }^{6} \chi$ denotes the phase accumulation for the electron moving between the two composite subsystems. Proper account for $\chi$ can describe the resonant tunneling. ${ }^{2}$ For simplicity, we hereafter set $\chi=1$, which is sensible for far off-resonance tunneling.

In practice of computing the $S$ matrix of the entire system, we start with $\mathbf{S}^{(1)}$ for the simple system of barrier-scattererbarrier. Applying the recursive technique described above and making use of Eqs. (1) and (2), we obtain

where $T_{i, j} \equiv T_{i j}=\left|S_{i j}\right|^{2} . \widetilde{\mathbf{K}}^{(1)}$ is a $N \times 1$ matrix with elements $\widetilde{K}_{n}^{(1)}=T_{2 n+1,1}+T_{2 n+2,1}$. Substituting the formal solution of Eq. (5), $\widetilde{\mathbf{J}}_{B}=(2 \mathbf{I}-\widetilde{\mathbf{T}})^{-} \widetilde{\mathbf{K}}^{(1)} J_{L}$, into the expression of the

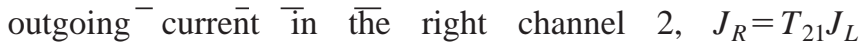
$+\widetilde{\mathbf{K}}^{(2)} \widetilde{\mathbf{J}}_{B}$, we obtain the effective tunneling probability under the momentum randomization boundary condition

$$
\widetilde{T}_{\text {eff }} \equiv J_{R} / J_{L}=T_{21}+\widetilde{\mathbf{K}}^{(2)}(2 \mathbf{I}-\widetilde{\mathbf{T}})^{-1} \widetilde{\mathbf{K}}^{(1)},
$$

where $\widetilde{\mathbf{K}}^{(2)}$ is an $1 \times N$ matrix with elements $\widetilde{K}_{n}^{(2)}=T_{2,2 n+1}$ $+T_{2,2 n+2}$.

Backscattering-free boundary condition. To elucidate this boundary condition, let us consider in more detail the scattering on the dephasing scatterer (e.g., the $n$th one), see Fig. 2. The scattering matrix $\mathbf{S}_{\epsilon}$ of Eq. (2) relates the outgoing amplitudes $\mathbf{a}_{n}^{\prime}=\left(a_{2 n+1}^{\prime}, a_{2 n+2}^{T}, b_{2 n+1}^{\prime}, b_{2 n+2}^{\prime}\right)^{T}$ to the incoming amplitūes $\mathbf{a}_{n}=\left(a_{2 n+1}, a_{2 n+2}, b_{2 n+1}, b_{2 n+2}\right)^{T}$ in terms of $\mathbf{a}_{n}^{\prime}=\mathbf{S}_{\epsilon} \mathbf{a}_{n}$. It is easy to check that the unitary property of $\mathbf{S}_{\epsilon}{ }$ and $\bar{i}$ s specific structure would lead to the conserving relations

$$
\left|a_{2 n+1}^{\prime}\right|^{2}+\left|b_{2 n+2}^{\prime}\right|^{2}=\left|a_{2 n+2}\right|^{2}+\left|b_{2 n+1}\right|^{2}
$$

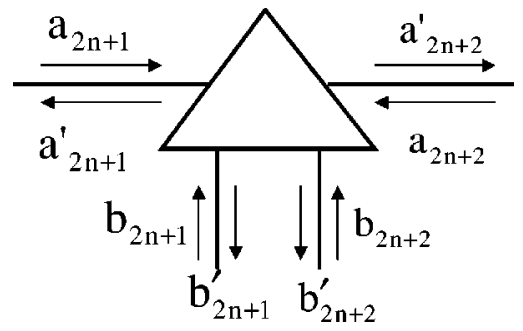

FIG. 2. Schematic diagram for the scattering on the $n$th dephasing scatterer. The outgoing amplitudes are connected with the incoming amplitudes via $\mathbf{a}_{n}^{\prime}=\mathbf{S}_{\epsilon} \mathbf{a}_{n}$, see text for more details. 


$$
\left|a_{2 n+2}^{\prime}\right|^{2}+\left|b_{2 n+1}^{\prime}\right|^{2}=\left|a_{2 n+1}\right|^{2}+\left|b_{2 n+2}\right|^{2} .
$$

Now we make use of the pure dephasing condition: $\left|a_{2 n+1}\right|^{2}=\left|a_{2 n+2}^{\prime}\right|^{2}$ and $\left|a_{2 n+2}\right|^{2}=\left|a_{2 n+1}^{\prime}\right|^{2}$. Its physical meaning is quite clear by noting that the phase breaking only randomizes the phases of the forward and backward going waves, but does not cause any reflections. Because of this nature, we term it as a backscattering-free boundary condition, which straightforwardly leads to

$$
\begin{aligned}
& \left|b_{2 n+1}^{\prime}\right|^{2}=\left|b_{2 n+2}\right|^{2}, \\
& \left|b_{2 n+2}^{\prime}\right|^{2}=\left|b_{2 n+1}\right|^{2} .
\end{aligned}
$$

With this physical insight, we are ready to derive the effective tunneling probability under the present boundary condition. We denote $J_{n}=\left(j_{2 n+1}, j_{2 n+2}\right)^{T}$ for the injecting currents from the $n$th reservoir into the system via the $(2 n$ $+1)$ th and $(2 n+2)$ th channels, and $J_{n}^{\prime}=\left(j_{2 n+1}^{\prime}, j_{2 n+2}^{\prime}\right)^{T}$ for the currents being scattered into the reservoir from the system via the same channels. The pure dephasing boundary condition, now denoted as $j_{2 n+1}^{\prime}=j_{2 n+2}$, and $j_{2 n+2}^{\prime}=j_{2 n+1}$, can be expressed compactly for all the $N$ reservoirs as

$$
\mathbf{J}_{B}^{\prime}=\underline{\mathbf{B}} \mathbf{J}_{B},
$$

where $\mathbf{J}_{B}=\left(J_{1}, J_{2}, \ldots, J_{N}\right)^{T}, \mathbf{J}_{B}^{\prime}=\left(J_{1}^{\prime}, J_{2}^{\prime}, \ldots, J_{N}^{\prime}\right)^{T}$, and $\mathbf{B}=\mathbf{I} \otimes \boldsymbol{\sigma} . \boldsymbol{\sigma}$ is a $2 \times 2$ matrix with elements $\sigma_{i j}=1-\delta_{i j}$. $\bar{A}$ gain, $\overline{\text { a }}$ simple current counting leads to

$$
\begin{gathered}
J_{R}=T_{21} J_{L}+\underline{\mathbf{K}^{(2)} \mathbf{J}_{B},} \\
\mathbf{J}_{B}^{\prime}=\underline{\mathbf{K}^{(1)} J_{L}}+\underline{\mathbf{T} \mathbf{J}_{B} .}
\end{gathered}
$$

Here, $\mathbf{K}^{(1)}$ is a $2 N \times 1$ matrix with elements $T_{n 1}$, and $\mathbf{K}^{(2)}$ an $1 \times 2 \bar{N}$ matrix with elements $T_{2 n}$, with $n=3,4, \ldots, \overline{2 N}+2$. T is a $2 N \times 2 N$ matrix with elements $T_{m n}$, with $m, n$ $\equiv 3,4, \ldots, 2 N+2$. Straightforwardly, by substituting Eq. (9) into Eq. (10) an elegant expression is obtained for the effective tunneling probability,

$$
T_{\mathrm{eff}}=J_{R} / J_{L}=T_{21}+\underline{\mathbf{K}}^{(2)}(\underline{\mathbf{B}}-\underline{\mathbf{T}})^{-1} \underline{\mathbf{K}}^{(1)} .
$$

This equation is formally similar to Eq. (6). Both Eqs. (6) and (11) contain a common coherent term (the first one), and an incoherent term (the second one). However, they physically differentiate from each other. Below we detail our discussions on their difference by focusing on the special case of one dephasing scatterer.

In the case of $N=1$, from Eq. (6) we easily obtain

$$
\begin{aligned}
\widetilde{T}_{\text {eff }} & =T_{21}+\left(T_{23}+T_{24}\right)\left(2-\widetilde{T}_{11}\right)^{-1}\left(T_{13}+T_{14}\right) \\
& =T_{21}+S_{b} S_{f} /\left(S_{b}+S_{f}\right) .
\end{aligned}
$$

Here, following Büttiker, ${ }^{1,2}$ the incoherent backward and forward scattering probabilities are introduced: $S_{b}=T_{13}+T_{14}$ and $S_{f}=T_{23}+T_{24}$. In deriving Eq. (12), the symmetry $T_{m n}$ $=T_{n m}$ and the sum rule $\Sigma_{m} T_{m n}=1$ have been used. On the other hand, for $N=1$, Eq. (11) leads to

$$
\begin{aligned}
T_{\text {eff }}= & T_{21}+\left[\left(T_{44} T_{23} T_{31}+T_{33} T_{24} T_{41}\right)\right. \\
& \left.+\left(1-T_{34}\right)\left(T_{23} T_{41}+T_{24} T_{31}\right)\right] / Z,
\end{aligned}
$$

where $Z=\left(1-T_{34}\right)^{2}-T_{33} T_{44}$.

Further, in the completely incoherent regime $(\epsilon=1)$, $T_{13}=T_{1} \equiv\left|t_{1}\right|^{2}, \quad T_{24}=T_{2} \equiv\left|t_{2}\right|^{2}, \quad T_{33}=1-T_{1}, \quad$ and $T_{44}=1$ $-T_{2}$. Other $T_{m n}$ in Eqs. (12) and (13) are zeros. In this regime, Eq. (12) reduces to ${ }^{2}$

$$
\widetilde{T}_{\text {eff }}=\left[\frac{1}{T_{1}}+\frac{1}{T_{2}}\right]^{-1},
$$

whereas Eq. (13) gives rise to ${ }^{19}$

$$
T_{\mathrm{eff}}=\left[\frac{1}{T_{1}}+\frac{1}{T_{2}}-1\right]^{-1} .
$$

The latter Eq. (15) can also be obtained by a rather simple treatment based on incoherent multiple reflections and transmissions through two barriers. ${ }^{24}$ The interesting difference between Eqs. (14) and (15) can be highlighted as follows. It is well known that the two-terminal resistance is related to the effective total transmission probability $T_{\text {tot }}$ (i.e., $\widetilde{T}_{\text {eff }}$ and $\left.T_{\text {eff }}\right)$ via the Laudauer formula, ${ }^{25} \mathcal{R}_{\text {tot }}=\left(h / e^{2}\right)\left(1 / T_{\text {tot }}\right)$. From Eq. (14) the system resistance can be expressed as

$$
\widetilde{\mathcal{R}}_{\text {tot }}=\mathcal{R}_{1}+\mathcal{R}_{2}+\mathcal{R}_{c}+\mathcal{R}_{s} .
$$

In this decomposed form, $\mathcal{R}_{j}=\left(h / e^{2}\right)\left(1-T_{j}\right) / T_{j}(j=1,2)$ is the Landauer resistance for the $j$ th conductor, $\mathcal{R}_{c}=h / e^{2}$ is the so-called contact resistance rooted in the two-terminal configuration (measurement). ${ }^{6,26}$ Interestingly, the dephasing scatterer contributes a constant resistance $\mathcal{R}_{s}=h / e^{2}$ in the completely incoherent regime, due to the momentum randomization. On the other hand, from Eq. (15) we have

$$
\mathcal{R}_{\text {tot }}=\mathcal{R}_{1}+\mathcal{R}_{2}+\mathcal{R}_{c} \text {. }
$$

We see that under the boundary condition Eq. (9) the dephasing scatterer only plays a phase-breaking role. As a result, the Landauer resistances are connected in series in a purely classical way, and the dephasing source does not cause additional resistance.

Interestingly, the backscattering-free nature on the dephasing scatterer can be further elucidated by considering the simple transmission through only a dephasing scatterer (i.e., with no tunnel barriers), with an arbitrary strength of incoherence. In this case, the relevant transmission coefficients are: $T_{21}=T_{34}=1-\epsilon, T_{13}=T_{24}=\epsilon, \quad T_{33}=T_{44}=T_{14}$ $=T_{23}=0$. Accordingly, Eq. (12) gives rise to $\widetilde{T}_{\text {eff }}=1-\epsilon / 2$, whereas Eq. (13) leads to $T_{\text {eff }}=1$. This result clearly shows the distinct nature of the two dephasing models: one causes backscattering, another is backscattering free.

For two resistors connected in partial coherence $(0<\epsilon$ $<1)$, the simple sum rule of the individual resistances as Eqs. (16) and (17) breaks down. However, the resistance difference $\Delta \mathcal{R}=\left(h / e^{2}\right)\left(1 / \widetilde{T}_{\text {eff }}-1 / T_{\text {eff }}\right)$ based on Eqs. (12) and (13) is a proper quantity to characterize the additional resistance caused by the dephasing scatterer under the the momentum randomization boundary condition. Figure 3 


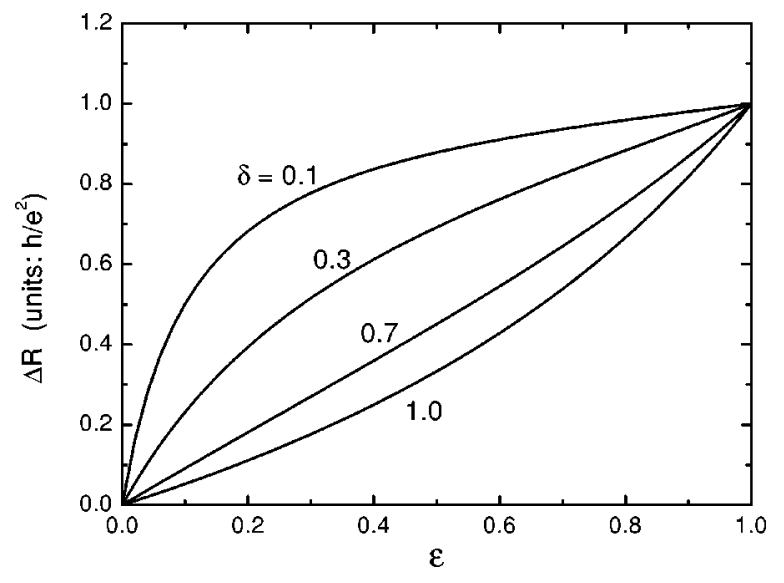

FIG. 3. Additional resistance caused by momentum randomization on the dephasing scatterer. Here the special case of two identical resistors connected in partial coherence is demonstrated to show the interplay between dephasing and tunneling. $\delta$ characterizes the tunneling strength through the individual resistor, i.e., $T_{1}$ $=T_{2}=\delta$.

shows $\Delta \mathcal{R}$ as a function of the incoherence strength $\epsilon$. In general, the interplay between the backscattering on the dephasing scatterer and the tunneling through the individual barriers leads to $\Delta \mathcal{R}(\epsilon)$ depending on the barrier-tunneling strength as shown in Fig. 3.

To further illustrate the application of Eqs. (6) and (11) in combination with the recursive rule of Eq. (3), we briefly present results for tunneling in the presence of multiple phase-breaking scatterers. For clarity, Fig. 4 shows the relative tunneling probabilities versus the incoherence strength, i.e., $T_{\text {eff }}(\epsilon) / T_{\text {eff }}(\epsilon=0)$ and $\widetilde{T}_{\text {eff }}(\epsilon) / \widetilde{T}_{\text {eff }}(\epsilon=0)$, by solid and dashed curves, respectively. In the weak tunneling regime ( $\delta=0.1$ ) shown in Fig. 4(a), dephasing enhances the tunnel-
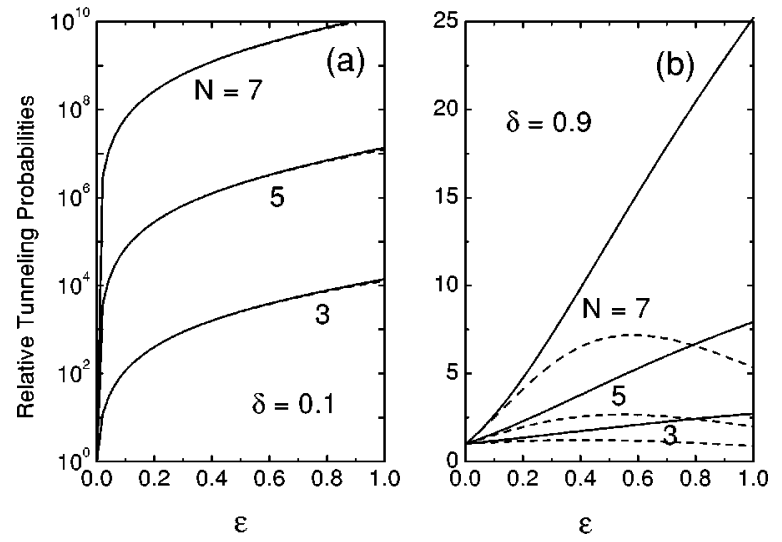

FIG. 4. Partially coherent tunneling through $N+1$ identical barriers (i.e., $T_{j}=\delta, j=1,2, \ldots, N+1$ ). The tunneling probabilities, $T_{\text {eff }}(\epsilon)$ [Eq. (11)] for the pure-dephasing boundary condition and $\widetilde{T}_{\text {eff }}(\epsilon)$ [Eq. (6)] for the momentum-randomization boundary condition, are demonstrated at relative values with respect to their coherent counterparts, in terms of $T_{\text {eff }}(\epsilon) / T_{\text {eff }}(\epsilon=0)$ (solid curve) and $\widetilde{T}_{\text {eff }}(\epsilon) / T_{\text {eff }}(\epsilon=0)$ (dashed curve), respectively. The weak (a) and strong (b) tunneling limits are plotted to highlight the similarity and difference between the two dephasing models in different regimes.

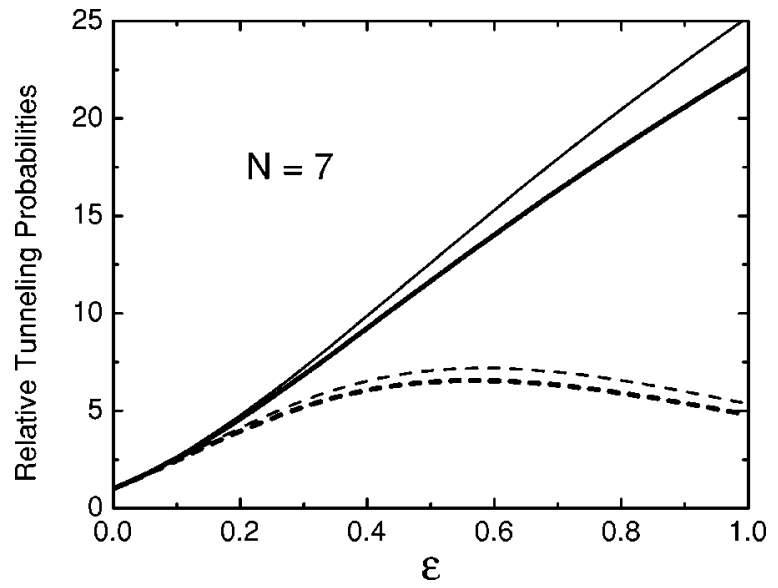

FIG. 5. Effects of nonuniformity on relative tunneling probability demonstrated for an $N=7$ case with individual values of tunnel $\delta$ parameter being chosen randomly around 0.9 . The solid and dashed curves are for the pure-dephasing and momentumrandomization boundary conditions, respectively. For comparision, those of the corresponding uniform (thin curves with all $\delta=0.9$ ) systems are also plotted against the nonuniform (thick curves; see text for values of $\delta_{j}$ ) counterparts.

ing remarkably, and the two dephasing models, i.e., Eqs. (6) and (11), give almost the same results. However, in the strong tunneling regime ( $\delta=0.9$ ) shown in Fig. 4(b), the two dephasing models give noticeably different results. More interestingly, the turnover behavior predicted by Eq. (6), which was paid special attention by Büttiker, ${ }^{1}$ does not occur in the pure dephasing model of Eq. (11). Therefore, the turnover behavior appears as a consequence of competition between the incoherent tunneling and the dephasing-induced backscattering. It disappears in the pure-dephasing (backscattering-free) limit.

Despite the above numerical demonstrations made for a uniform system, the basic features regarding the backscattering effect would be retained in the nonuniform system. The qualitative difference shown in Fig. 4 is rooted in the two different boundary conditions associated with the dephasing reservoirs, being irrelevant to the nonuniformity. This conclusion is demonstrated in Fig. 5, where the nonuniform system is simulated by varying the tunneling strength through each barrier around $\delta=0.9$ randomly in the range of 0.05 . The eight nonuniform tunneling parameters, 0.86, 0.92, 0.89, $0.94,0.93,0.85,0.86$, and 0.94 , are used for demonstrative $N=7$ systems in this figure. Besides the aforementioned features regarding the backscattering effects, Fig. 5 also shows that the nonuniformity decreases tunneling probability in either pure-dephasing or momentum-randomization boundary condition.

Generally speaking, the Büttiker dephasing model is a phenomenological approach, which is valuable for its simplicity and the strong describing ability. Its microscopic basis has been exploited on the basis of realistic electron-phonon scattering mechanism. ${ }^{3-5}$ Recently, we have demonstrated that, as far as the stationary tranport or transfer rate processes is concerned, the Büttiker scattering matrix approach can give similar results as the microscopic reduced density- 
matrix formalism. ${ }^{27}$ In the latter case, stochastic reservoir models are used to simulate the dissipations such as in the long-range charge transfer in chemical/biological systems. In this context, the pure-dephasing model in the complete incoherence limit $(\epsilon=1)$ corresponds to the sequential hopping mechanism proposed by the charge-transfer community. ${ }^{28}$ Other physical context in connection with the puredephasing model can be referred to the high-magnetic field transport in ballistic junctions. ${ }^{29}$ Physically, the pure dephasing model should be appropriate if the separation between the tunnel barriers is short compared to the inelastic length but longer than or comparable to the phase-breaking length.

In summary, we have presented a unified treatment for phase-breaking tunneling based on the Büttiker model.
Simple adoption of two types of boundary conditions can clear up the distinct natures of two dephasing models. The derived explicit expressions in combination with the recursive rules can be conveniently applied to tunneling through multibarrier systems with arbitrary strengths of incoherence.

Support from the Research Grants Council of the Hong Kong Government and the National Natural Science Foundation of China is gratefully acknowledged. X.Q.L. specially thanks for the distinguished young researchers program from CAS (Chinese Academy of Sciences). Finally, we sincerely thank Professor M. Büttiker for the kind communication after this paper appeared as e-print cond-mat/0106439.
${ }^{1}$ M. Büttiker, Phys. Rev. B 33, 3020 (1986).

${ }^{2}$ M. Büttiker, IBM J. Res. Dev. 32, 63 (1988).

${ }^{3}$ M.J. McLennan, Y. Lee, and S. Datta, Phys. Rev. B 43, 13846 (1991).

${ }^{4}$ S. Hershfield, Phys. Rev. B 43, 11586 (1991).

${ }^{5}$ S. Datta, Phys. Rev. B 46, 9493 (1992).

${ }^{6} \mathrm{~S}$. Datta, Electronic Transport in Mesoscopic Systems (Cambridge University Press, New York, 1995).

${ }^{7}$ D.K. Ferry and S.M. Goodnick, Transport in Nanostructures (Cambridge University Press, New York, 1997).

${ }^{8}$ J.L. D'Amato and H.M. Pastawski, Phys. Rev. B 41, 7411 (1990).

${ }^{9}$ M. Schreiber and M. Maschke, Philos. Mag. 65, 639 (1992).

${ }^{10}$ K. Maschke and M. Schreiber, Phys. Rev. B 49, 2295 (1994).

${ }^{11}$ F. Gagel and K. Maschke, Phys. Rev. B 54, 13885 (1996).

${ }^{12}$ T. Ando, Surf. Sci. 361/362, 270 (1996).

${ }^{13}$ N.A. Mortensen, A.-P. Jauho, and K. Flensberg, Superlattices Microstruct. 28, 67 (2000).

${ }^{14}$ M.T. Liu and C.S. Chu, Phys. Rev. B 61, 7645 (2000).

${ }^{15}$ C.W.J. Beenakker and M. Büttiker, Phys. Rev. B 46, 1889 (1992).

${ }^{16}$ T. Christen and M. Büttiker, Phys. Rev. B 55, R1946 (1997).

${ }^{17}$ C. Texier and M. Büttiker, Phys. Rev. B 62, 7454 (2000).

${ }^{18}$ Ya.M. Blanter and M. Büttiker, Phys. Rep. 336, 1 (2000).

${ }^{19}$ M. Büttiker, in Resonant Tunneling in Semiconductors: Physics and Applications, edited by L.L. Chang, E.E. Mendez, and C. Tejedor (Plenum, New York, 1991), p. 213.

${ }^{20}$ M. Büttiker, in Proceedings of the 13th International Conference on Noise in Physical Systems and 1/f Fluctuations, edited by V. Bareikis and R. Katilius (World Scientific, Singapore, 1995), p. 35 .

${ }^{21}$ See Ref. 6, pp. 129-132.

${ }^{22}$ I. Knittle, F. Gagel, and M. Schreiber, Phys. Rev. B 60, 916 (1999).

${ }^{23}$ E. Merzbacher, Quantum Mechanics, 3rd ed. (Wiley, New York, 1998).

${ }^{24}$ See Ref. 6, p. 64.

${ }^{25}$ R. Landauer, IBM J. Res. Dev. 1, 223 (1957); Philos. Mag. 21, 863 (1970).

${ }^{26}$ Y. Imry, in Directions in Condensed Matter Physics: Memorial Volume in Honor of Prof. S.K. Ma, edited by G. Grinstein and G. Mazenko (World Scientific, Singapore, 1986), pp. 101-163.

${ }^{27}$ X.Q. Li and Y.J. Yan, J. Chem. Phys. 115, 4169 (2001).

${ }^{28}$ X.Q. Li, H. Zhang, and Y.J. Yan, J. Phys. Chem. 105, 9563 (2001), and references therein for the hopping mechanism for long-range charge transfer in DNA.

${ }^{29}$ M. Büttiker, Phys. Rev. B 38, 12724 (1988); G. Kirczenow, Solid State Commun. 71, 469 (1989). 